\title{
Mobile Technologies and Their Role in the Development of Musical Ear
}

\author{
Gorbunova I. B. and Goncharova M. S. ${ }^{1}$ \\ ${ }^{1}$ Herzen State Pedagogical University of Russia, St. Petersburg, Russia
}

\begin{abstract}
The processes of informatization transform the environment of professional activity of the contemporary musician, form new educational needs of students of various musical specialties, cause changes in the activity of the teacher-musician. This is due, in particular, to the use of digital educational resources, the widespread introduction of techniques and methods of network interaction and modern music and computer technologies (MCT), which have a wide range of capabilities. These possibilities are truly limitless, they allow you to find new artistic worlds. One of the actual problems of musicology is the study of the processes associated with the spread of information culture and their influence on the formation of the contemporary musician, in particular the formation of musical ear. The authors of the article analyze the possibilities of mobile technologies in the field of development of musical hearing of children's musical schools and children's schools of arts. Based on the results of many years of practice of teaching solfeggio with the use of mobile technologies and the conducted pedagogical experiment, the authors conclude about the high educational potential of cloud-oriented technologies in the system of contemporary musical education. Mobile technologies can significantly expand the forms and methods of teaching solfeggio. The authors of the article note the need for further scientific and pedagogical research in this area related to teaching with the use of modern mobile technologies, the development of training programs and professional retraining of teachers of musical and theoretical disciplines.
\end{abstract}

Keywords: mobile technologies, musical ear, music computer technologies (MCT), solfeggio, music education

\section{Introduction}

The processes of Informatization transform the environment of professional activity of the modern musician, form new educational needs of students of various musical specialties, cause changes in the activity of the teacher-musician. This is due, in particular, to the use of digital educational resources, the widespread introduction of techniques and methods of network interaction and modern music computer technologies (MCT), which have a wide range of capabilities [1], [2], [7]. The development of a new educational direction - music and computer technology as a learning and creative environment in General, professional and special (inclusive) musical education began in the Herzen State Pedagogical University of Russia in 2002 when, under the guidance of Prof., Dr. of Pedagogical Sciences Irina B. Gorbunova were created Educational and Methodical laboratory 'Music Computer Technologies'. The laboratory has organized academic conferences and seminars, creative competitions and concerts. Developed, licensed and implemented in the pedagogical process of professional and educational profile of bachelors of art education (050610 "Music Computer Technologies"), the program of master's training (050610M "Music Computer Technology in Education"), a number of specialties for teachers and musicians are trained in refresher courses.

These possibilities are truly limitless, they allow you to find new artistic worlds [6]. One of the actual problems of musicology is the study of the processes associated with the spread of information culture and their influence on the formation of the contemporary musician [3], [4], [5].

As part of the dynamically developing information technology, mobile technologies combine tools and methods that allow working with digital data through the use of mobile devices connected to information networks: laptops, smartphones, tablets. For those who are accustomed to the standard computer keyboard, it is possible to connect it to the tablet via USB output, Bluetooth or Wi-Fi Direct system. MIDI keyboard is 
connected in the same way. Tablets can be connected to other devices: external drives, monitors, printers, scanners, etc.

An important advantage of mobile technologies is the ability to provide rapid access to cloud-based educational services and resources. The main purpose of cloud computing and cloud services, as well as a number of Web applications, is to provide the user with access to all their data or software from any digital medium. Owners of tablets on Android and iOS (Apple) are familiar with the concept of cloud storage, as Google-Disk and iCloud services allow you to store all sorts of information (photos, contacts, video, audio, applications), as well as synchronize your data from tablets and smartphones with the cloud.

\section{Mobile technologies in education}

Mobile technologies will change the quality of interaction between teachers and students, providing access to shared resources and exchange of information at any time. This will contribute to the testing and implementation of innovative teaching methods, improve the organization and automation of processes, as well as open up fundamentally new economic opportunities. In the field of development of musical ear mobile technologies can significantly expand the forms and methods of teaching solfeggio [8], so it is important to identify their educational potential.

Currently, there is a growing and widespread enthusiasm for tablets, which offers an infinite number of easily accessible and exciting Internet services and applications. For today's students tablets are not something unusual and complex, because due to the high degree of intuitiveness of the interface they can be used without learning the principles of operation of these devices. Tablet technologies can be used as a completely new, different in form and content platform for more entertaining and interactive learning [8], [9]. In this regard, an important task is the development of teachers-musicians practical teaching skills with the involvement of mobile technologies. The introduction of a new innovative form of learning will require special research and adaptation of learning materials to mobile devices. The need to adapt the material due to the fact that the content on the mobile platform is served in short blocks, should be extremely straightforward and simple, but without prejudice to the content and taking into account the peculiarities of music classes.

Unfortunately, not every teacher today owns the means of high-tech educational environment. All this poses fundamentally new challenges for the education system that require new technological solutions and the development of pedagogical conditions for their effective use, the search for scientific approaches to the organization of the educational process in music education.

To solve these problems, first of all, it is necessary to get acquainted with the quality and features of the widespread and popular mobile applications that are used for the development of musical hearing.

In the field of development of musical ear mobile technologies can significantly expand the forms and methods of teaching solfeggio [10], so it is important to identify their educational potential.

At the moment, there are a huge number of training applications aimed at the development of various aspects of musical literacy, musical hearing and thinking. Most of the well-known mobile applications are designed to acquaint with musical notation and the basic elements of the musical language, to promote the development of harmony hearing and sense of rhythm, to control the accuracy of pitch intonation, to expand the auditory baggage, in short, to be a simulator for the development of musical and auditory skills.

Many students use a variety of applications to work with audio (trim ringtone, connect melodies to each other, etc.) or video (video conversion, processing, the ability to make a video clip from photos , picking up music to it; for example: Photo Editor Pro, Song Editor, Magisto), as well as simple applications (for example: Drum Pads 24 or Music Maker Jam) for composing music in different modern styles (Drum'n'bass, Dubstep, Electronic, Trip Hip Hop, etc.). These applications contain professional Studio samples, pads (synthesized sounds) that allow you to create and record your own improvisations, as well as share them with friends. 
An important stage in the formation of musical abilities at the initial stage of training is the development of singing skills and elementary intonation. Here can help sounding lullabies, nursery rhymes, Proverbs, tongue twisters, etc., which enrich the vocabulary, develop articulation apparatus, phonemic hearing, etc. you can give an example of the application of Nursery Rhymes and Tongue Twisters on cardboard, which in an interactive form will help students cope with articulation problems.

The application Absolute hearing-2 has a huge number of exercises focused on the development of hearing: compare the hearing intervals, determine the frets and chords, record melodic dictations, develop a sense of metrorhythm, the presence of a microphone on the tablet allows you to record the sung intervals and chords. The undoubted advantage is that the training of hearing and the form of knowledge testing turns into an exciting process, and it is very welcome by students. The ability to create your own exercises allows teachers to customize each student's learning at an individual pace and level of difficulty. The presence of a theoretical section in the application (the author of the articles "Intervals", "Chords", "Harmony", etc. is Mary S. Goncharova) provides the student with the information necessary to perform interactive exercises.

Music First has created an interesting app for music teachers and students based on cloud solutions. The site contains a variety of music tutorials that were previously only available on DVD (sequencers; learning to play various instruments; programs for training hearing; music editors; as well as programs that introduce the basics of music theory and history, etc.). All actions of students and teachers are stored and synchronized in the cloud, allowing teachers to conduct a thorough monitoring and evaluation of the learning process at any time. This is an extremely useful resource for distance music education.

A variety of exercises that develop singing skills and expand the vocal range are contained in the Vocalist Lite application. You can import the necessary melodies into the application, sing, and the screen instantly displays comments if the wrong note was sung. This application is convenient to use for self - test-for example, before passing the learned numbers on solfeggio.

For the development of creative abilities in the classroom solfeggio convenient to use the application Chordbot Lite, with which students can make their own arrangement to the previously recorded dictation or harmonization to the learned melody. This form of work is particularly suitable for performers on wind and stringed instruments who have difficulty playing the piano when performing creative tasks on solfeggio.

Owners of iPad, iPhone, iPod in standard applications have GarageBand program, which is a digital audio workstation and sequencer for recording and playback of multi-channel audio. This app has over a hundred virtual instruments. You can play these instruments using the virtual keyboard, but you can also connect a MIDI keyboard. An important feature of this application is the section "music Lessons", which allows you to download audio and video lessons of playing the piano or guitar, and comments and tips make it possible to quickly improve the skills of the game. At the same time, students independently use a variety of educational applications, which helps them prepare for various subjects of the musical-theoretical cycle.

Applications Walk Band for Android, GarageBand for iOS allow you to create a whole orchestra of different instruments, which is a great alternative to noise orchestras. This form of work is suitable for learning new rhythmic patterns. Rhythm is a leading component of the formation and organization of musical tissue in General, so a variety of exercises will only improve the mastery of the elements of the metrorhythm. Ensemble play is a comprehensive method of development of students, it provides an opportunity for mutual learning and mutual education, as a fertile ground for the birth of a new musical sound in an atmosphere of cooperation. A well-chosen repertoire of ensemble music expands the horizons of what children know in music, replenishes the Fund of their auditory impressions, which plays an active role in the process of formation and development of musical thinking and intelligence.

The NotateMe Now app, with its recognition feature, allows you to quickly record sheet music in the usual handwriting way with a stylus (e-pen) or with a touch of a finger: the tablet simulates a sheet of paper, and the epen acts as a pen or pencil. 
In some applications, the multichannel recording feature opens up a huge field of musical creativity and various forms of work with musical fragments for students. You can write small scores, preinstrumental them to cut and combine different pieces, record their own works, to improvise. The material is recorded in MIDI format, created or imported MIDI files can be edited in the piano roll or using the stave. Application functionality, such as MidiSheetMusic, allows you to import the finished MIDI material into PDF format (for printing). Also, the work can be listened to, on the screen at the same time marked sounding notes on the stave and virtual piano keyboard.

A professional development program "Tablet and cloud-oriented technologies in contemporary musical education" for teachers of children's musical schools and children's schools of arts: some methodical aspects

We have developed a professional development program for teachers of children's musical schools and children's schools of arts "Tablet and cloud-oriented technologies in contemporary musical education". Fragments of classes using these technologies in the teaching of various music disciplines in the school can be seen here: https://youtu.be/rL-G5Pi80rs.

It is important to note that the use of mobile applications in the classroom for ear training in children's musical schools and children's schools of arts can be combined with traditional forms of learning. For example, when studying the theme "Reduced triads" on the piano department in the fifth grade (the full program of training in the system of primary professional music education in the children's musical schools and children's schools of arts in Russia is nine years) you can use the following options of tasks:

1) Compose a 4, 6 or 8-beat melody in harmonic minor using the Maestro app. This application is a simplified version of the musical set of one-voice melody with a choice of key, instrument, etc.

2) Import the composed melody into MIDI format and open it through the WalkBand application (for recording the second voice or harmonisation and subsequent instrumentation, adding a rhythmic accompaniment) or through the Chordbot Lite application (for auto-arrangement of the selected harmony also with the use of reduced triad).

3) Record the performance of the composed melody with accompaniment using multi-channel recording in the WalkBand and export to MP3 format.

4) Listen to several excerpts (for example, the part of Naina from Glinka's Opera Ruslan and Lyudmila, the Minuet of J. Vekerlen, the Aria of the Snow Maiden from the Opera Snow Maiden by N.A. Rimsky-Korsakov, etc.) on the YouTube channel or in the Perfect Piano application (on the scores in PDF format during listening the student sees the piano keyboard, on which the sounding keys are marked in real time). To determine which passage sounds the diminished triad or newts, to make them the screenshots and in the resulting drawings to celebrate these chords and intervals. The answer should be sent to the General class forum or to the cloud storage of homework or work in the classroom.

Having studied the available software and features of their perception by students, having practical experience in this area, we came to a number of conclusions. First of all, we note that the process of mobile learning includes the main pedagogical functions (motivational, information, function of management of educational activities, forming skills, controlling and correcting) and the possibility of information and telecommunication technologies to stimulate creative activity to the study of the material and the search for an answer, flexibility, adaptability and taking into account the cognitive capabilities of students, training, the ability to take different ways of answering.

There are a number of undeniable advantages of using mobile technologies in education and, in particular, in the development of musical hearing and thinking. Note the most, in our opinion, significant.

At the initial stage of learning tablet computer is usually used as a replacement of e-books and textbooks. However, the possibilities of the electronic textbook in this case are greatly expanded: wireless Internet access using Wi-Fi technology opens the way to a huge knowledge base, for students becomes available to help the 
tutor and teacher (commenting, discussion, video, etc.). Thanks to access to the Internet, you can instantly search for the necessary information, update it, create a hyperlink, view materials online, listen to audio, view video, that is make the electronic textbook more interactive and multimedia. The teacher has the ability to create access for students to a huge music library. You can also create interactive servers with music quizzes and tests, which will be available to all students through the web-browser of the tablet.

\subsection{Access to learning tools, visibility and adequate forms of implementation of educational interaction}

The basis of any pedagogical process is access to learning tools, visibility and adequate forms of implementation of educational interaction. Thanks to the tablets, this is very easy to achieve. Programs come to life with a single touch to the screen, learning takes place in a completely new dimension (as you know, visualspatial methods of perception of musical material are best suited to achieve concentration). Handwriting connects motor memory to the learning process at a higher level than using a PC-keyboard.

Compact tablet allows you to use it in any conditions. The presence of a touch keyboard and a gesture interface, a silent set of book and music text allow users to focus in a crowded environment. In the process of private learning, you can use headphones, which allows students with different levels of knowledge to be in the same class, performing individually selected tasks.

A sufficiently powerful acoustic subsystem (or an additional portable speaker system like JBL Go) allows you to use the tablet as a music center or a recording device: repeatedly listen to a certain piece of the sounding work, adjust the volume, change the ratio of high and low frequencies, etc. in the lessons of solfeggio, you can sound the score, turn off certain instrumental parts, change the timbres, etc.

\section{Conclusion}

Mobile learning applications and simulators, based on the integration of various forms of activity, significantly enhance the development of musical hearing and thinking, since the understanding of the elements of the musical language occurs through sensations and visual representations, which in comparison with verbal communication has a more specific, simple and dynamic nature of perception [11]. Indisputable effectiveness on the solfeggio lessons have interactive creative exercises with the use of mobile technology. Interactive exercises activate auditory attention, train musical memory and various aspects of musical hearing, develop taste and observation, imaginative and emotional thinking.

The use of mobile devices creates the need to organize the educational process with an optimal balance between the established traditions and new information technologies in the teaching of music. At the same time, the process of mastering knowledge is translated into the plane of finding the necessary information, repetition and cramming turn into an exciting creative process, and the study of solfeggio becomes one of the ways of contemporary musical communication.

\section{References}

[1] Goncharova M. S., Gorbunova I. B. (2016). Tablet (mobile) technology in professional music education, Mediamusic, no. 6. [Online]. 21(3), pp. 3-5. Available: http://mediamusic-journal.com/Issues

[2] Gorbunova I.B. "Electronic Musical Instruments: to the Problem of Formation of Performance Mastery", Int'l Conference Proceedings, Budapest, Hungary, 2018, pp. 23-28.

[3] Gorbunova I.B. "New Tool for a Musician", ICASET-18, AS-BES-18, EEHIS-18 International Conference Proceedings, Paris, France, 2018, pp. 144 - 149.

[4] Gorbunova I.B. "Electronic Musical Instruments: to the Problem of Formation of Performance Mastery", Int'l Conference Proceedings, Budapest, Hungary, 2018, pp. 23-28.

[5] Gorbunova I., Govorova A. (2018) Music Computer Technologies in Informatics and Music Studies at Schools for Children with Deep Visual Impairments: From the Experience. In: Pozdniakov S., Dagienè V. (eds) Informatics in 
Schools. Fundamentals of Computer Science and Software Engineering. ISSEP 2018. Lecture Notes in Computer Science, vo.l 11169. Springer, Cham

[6] Gorbunova I.B., Davletova K.B., and Tovpich I.O., "Electronic Musical Instruments in the General Music Education System", ICASET-18, ASBES-18, EEHIS-18 International Conference Proceedings, Paris, France, 2018, pp. 139-143.

[7] Gorbunova Irina B. and Hiner Hellene, Music Computer Technologies and Integrative Network Systems of Learning Music, The IIER International Conference, Oxford, UK, 2018, pp. 16-21.

[8] Gorbunova I. B., Pomazenkova M.S. Music Computer and Cloud-Oriented Technologies in Contemporary Musical Education. Scientific Opinion, no. 3-2, pp. 68-82, 2015.

[9] Gorbunova I. B., Pomazenkova M.S., and Tovpich I. O. Tablet and Music Computer Technologies in the System of Professional Music Education. Theory and Practice of Social Development, no. 8, pp. 211-219, 2015.

[10] Gorbunova I. B., Tovpich I. O. "Information educational environment as a resource of formation of information culture of participants of educational process in the school of the digital age", Theory and Practice of Social Development, no. 7, pp. 192-196, 2015.

[11] Gorbunova I.B., Zalivadny M.S. The Integrative Model for Semantic Space of Music: Perspectives of Unifying Musicology and Music Education, Music Scholarship, no. 4, pp. 48-57, 2018. 